\title{
Self-Wicking Nanowire Grids
}

Hui Wei ${ }^{1}$, Venkata P. Dandey ${ }^{1}$, Zhening Zhang ${ }^{1}$, Ashleigh Raczkowski ${ }^{1}$, Willam J. Rice ${ }^{1}$, Bridget Carragher $^{1,2}$ and Clinton S. Potter ${ }^{1,2}$

1. National Resource for Automated Molecular Microscopy, Simons Electron Microscopy Center, New York Structural Biology Center, NY.

2. Department of Biochemistry and Molecular Biophysics, Columbia University, New York, NY.

We have been developing a new type of grid for cryo electron microscopy (cryoEM) that is essentially "self-blotting" [1] and can be used in conjunction with a robotic device called Spotiton [2,3] that dispenses small volumes $(\sim 50 \mathrm{pL})$ of sample using a piezo electric nozzle. Nanowires can be grown on the copper surface of an EM grid using a simple chemical reaction. We usually use sandwich grids where one side is copper and the other is Rhodium (figure 1) so as to provide one smooth surface for adhering to the plastic substrate which can have either lacey or patterned holes, choice of carbon or gold film can be put on the plastic and then the plastic is removed to leave one with the desired substrate on the nanowire grid. When small volumes of sample are applied to the nanowire grids as soon as the sample comes into contact with the wires it is rapidly wicked away, leaving behind a thin film that is then rapidly plunged into liquid ethane to achieve vitrification.

We have used the nanowire grids to vitrify a wide variety of samples including Apoferritin, 20S proteasome, 70S E.Coli ribosome, hemagglutinin, aldolase, IgG antibody, HIV trimer complexes, membrane proteins in nanodiscs, insulin receptors, and glutamate dehydrogenase.

We will provide a detailed description of how we make these grids (figure 2) using a variety of support mesh and substrates. We will report on how the geometry of the grid and the local geometry of the substrate affects the wicking speed, ice thickness and distribution of particles in the holes [4].

\section{References:}

[1] Wei et al, Optimizing "self-wicking” nanowire grids. J Struct Biol. (2018).

[2] Razinkov et al, J Struct Biol. 195(2) (2016), p. 190.

[3] Dandey et al, Spotiton: New features and applications. J Struct Biol. (2018).

[4] (GM103310, OD019994-01) and the Agouron Institute (F00316). 


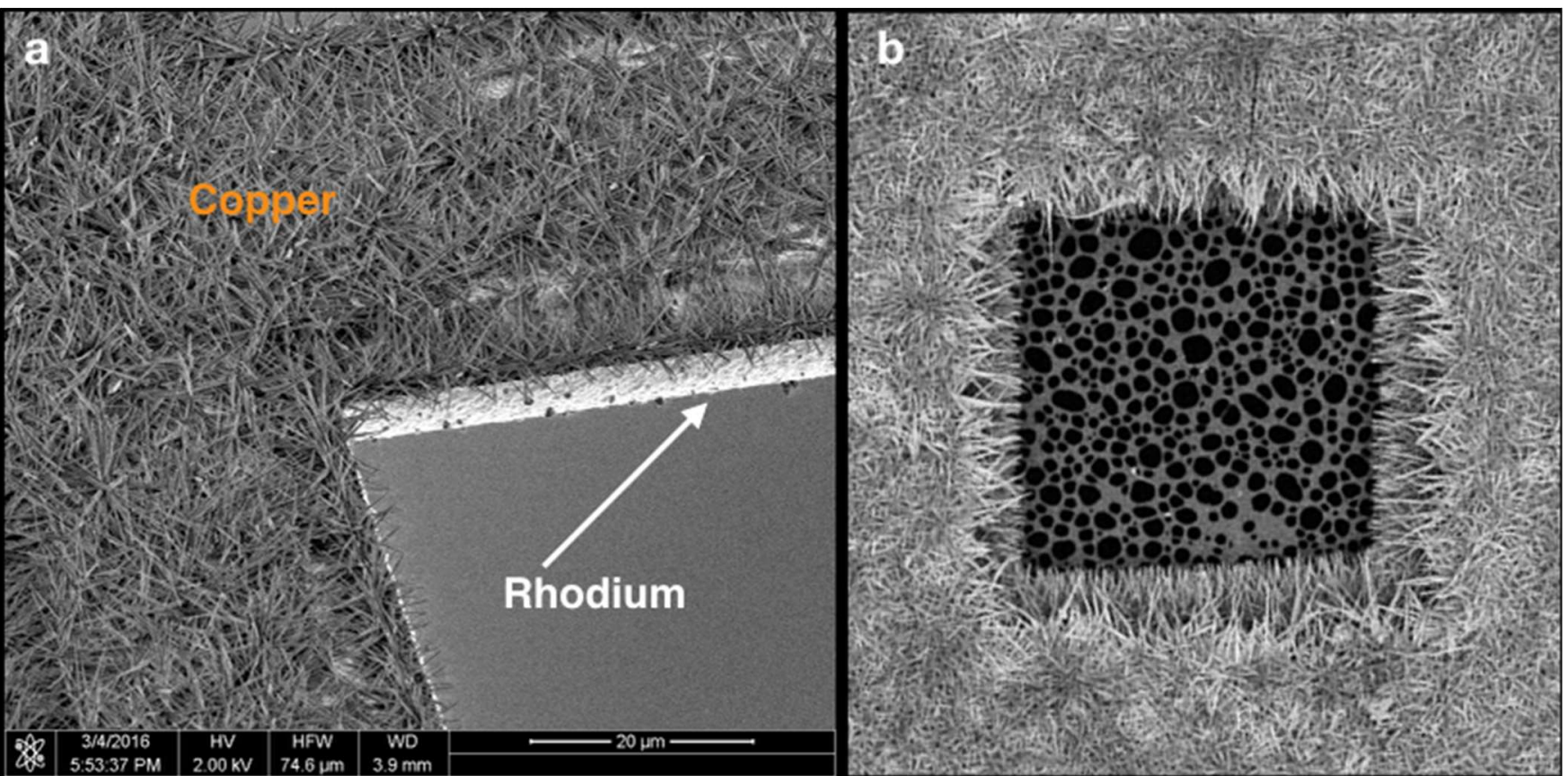

Figure 1. (a) Nanowires are grown on the surface of copper grid bars; the opposite surface, made of Rhodium, does not grow wires and provides a smooth surface for adhering substrate. (b) A lacey holey carbon substrate picked up onto the back side of the nanowire grid.

Materials and chemicals needed: $\mathrm{ddH}_{2} \mathrm{O}, 10 \mathrm{M} \mathrm{NaOH}, 0.52 \mathrm{M}$ ammonium persulfate solution, 300 mesh Copper/Rhodium Maxtaform Grids (EMS or Ted Pella), lacey plastic film or patterned plastic film, carbon rod (EMS, cat\#70210) or 0.20 mm diameter gold wire (EMS, cat\#73100).

1. Make fresh nano-wire growing solution: 2 parts dd $\mathrm{H}_{2} \mathrm{O}+1$ part $0.52 \mathrm{M} \mathrm{APS}+1$ part $10 \mathrm{M} \mathrm{NaOH}\left(500 \mathrm{ul} \mathrm{dd} \mathrm{H}_{2} \mathrm{O}+250 \mathrm{ul}\right.$ $0.52 \mathrm{M} \mathrm{APS}+250 \mathrm{ul} 10 \mathrm{M} \mathrm{NaOH})$, mix it well before use.

2. Float a 300 mesh $\mathrm{Cu} / \mathrm{Rh}$ grid on top of a 25 ul drop of nanowire solution sitting on parafilm, such that the copper side of the grid is facing down into the solution.

3. After 3 minutes, rinse the grid $2 x$ in sequential drops of $d d \mathrm{H}_{2} \mathrm{O}$.

4. Place the grid onto filter paper to dry.

5. Examine the grid under a light microscope at 500X magnification. Nanowires should be clearly observable on the copper surface (see figure $1 \mathrm{~b}$ ). The density of the nanowires will be related to the incubation time of the grid in the nanowire solution and this time can be adjusted to achieve the desired nanowire density.

6. Float holey plastic film (either lacey (Fukami and Adachi, 1965) or patterned (Marr et al., 2014)) onto a clean water surface in a glass container (about $12 \mathrm{~cm}$ in diameter).

7. Place nanowire grids, nanowire side up, onto the film, keeping a $\sim 2 \mathrm{~mm}$ gap between grids.

8. Place a piece of parchment paper on top of the film with grids and quickly lift the grids and film out of the water.

9. Lay the parchment paper with grids facing up on top of filter paper to dry with weights on the corners to prevent curling of the paper.

10. Coat a layer of $\sim 12 \mathrm{~nm}$ carbon film onto the top (plastic film side) of the grids. We used a Leica ACE600 carbon coater for this purpose.

11. Quickly dip ( 1 second) the coated grids into an acetone bath to remove the Lacey plastic film.

12. Place the grids in an oven at $80^{\circ} \mathrm{C}$ overnight before use which will help to prevent the carbon film from breaking.

For making nanowire grids with gold film, additional steps are as follows:

13. Evaporate a thin layer of gold, $\sim 10 \mathrm{~nm}$, onto the carbon substrate nanowire grids from step 12 . The nanowire side should be facing down while coating the gold film. We used an Edwards auto306 evaporator.

14. Remove the carbon film using a plasma cleaner with the nanowire side facing up. We used a Gatan Solarus model 950 plasma cleaner at full power for 5 minutes using an argon and oxygen gas mix.

Figure 2. Protocol for making nanowire grids. 